\title{
ADESÃO DE PACIENTES COM INSUFICIÊNCIA CARDÍACA À TERAPÊUTICA INSTITUIIDA
}

\author{
ADHERENCE OF PATIENTS WITH HEART \\ FAILURE TO THE ESTABLISHED THERAPY
}

\section{ADHESIÓN DE PACIENTES CON INSUFICIENCIA CARDÍACA A LA TERAPIA ESTABLECIDA}

\author{
Mailson Marques de Sousa ${ }^{1}$ \\ Rhaira Pereira Campos ${ }^{2}$ \\ Jacira dos Santos Oliveira ${ }^{3}$ \\ Simone Helena dos Santos Oliveira ${ }^{4}$
}

Como citar este artigo: Sousa MM, Campos RP, Oliveira JS, Oliveira SHS. Adesão de pacientes com insuficiência cardíaca à terapêutica instituída. Rev baiana enferm. 2019;33:e30442.

\begin{abstract}
Objetivo: analisar a associação entre as variáveis sociodemográficas e clínicas e a adesão de pacientes com insuficiência cardíaca ao tratamento, e avaliar a adesão desses pacientes ao tratamento. Método: estudo transversal realizado com 50 pacientes em seguimento ambulatorial. Utilizou-se um questionário composto por 10 itens para avaliar a adesão à terapêutica. Aplicou-se estatística descritiva, teste t de Student e ANOVA one way. Resultados: a média do escore de adesão ao tratamento foi de 13,36 $\pm 2,07$ pontos, sendo insatisfatória. Observou-se que as questões referentes à frequência do peso corporal e o controle na ingestão de líquidos apresentaram as menores proporções de respostas no escore de adesão. Conclusão: a análise da associação entre as variáveis sociodemográficas e clínicas e a adesão dos participantes com insuficiência cardíaca mostrou baixa adesão à terapêutica instituída.
\end{abstract}

Descritores: Insuficiência Cardíaca. Terapêutica. Cooperação do Paciente. Cooperação e Adesão ao tratamento. Enfermagem.

Objective: to analyze the relation between the socio-demographic and clinical variables and the adherence of patients with heart failure to the treatment, and to evaluate this adherence. Method: a cross-sectional study conducted with 50 outpatients. A 10-item questionnaire was used to assess adherence to therapy. Descriptive statistics, Student's t-test and one way ANOVA were used. Results: The mean adherence score was $13.36 \pm 2.07$ points, being unsatisfactory. It was observed that the questions regarding the frequency of body weight and the control of fluid intake had the lowest number of answers in the adherence score. Conclusion: the analysis of the relation between the socio-demographic and clinical variables and the adherence of the participants with heart failure showed low adherence to the established therapy.

Descriptors: Heart Failure. Therapy. Patient Cooperation. Cooperation and Adberence to Treatment. Nursing.

Objetivo: analizar la asociación entre las variables sociodemográficas y las clinicas y la adhesión al tratamiento de pacientes con insuficiencia cardiaca, además de evaluar dicha adhesión. Método: estudio transversal realizado con 50 pacientes en seguimiento ambulatorio. Se utilizó un cuestionario compuesto por 10 puntos para evaluar la adhesión a la terapia. Se aplicó estadística descriptiva, la prueba t de Student y ANOVA one way. Resultados: la

Enfermeiro. Mestre em Enfermagem. Universidade Federal da Paraíba. João Pessoa, Paraíba, Brasil. mailson ms@hotmail.com

Enfermeira. Pesquisadora independente. João Pessoa, Paraíba, Brasil.

Enfermeira. Doutora em Enfermagem. Professora da Universidade Federal da Paraíba. João Pessoa, Paraíba, Brasil.

Enfermeira. Doutora em Enfermagem. Professora da Universidade Federal da Paraíba. João Pessoa, Paraíba, Brasil. 
media del puntaje de adhesión al tratamiento fue de 13,36 2,07 puntos, y se la consideró satisfactoria. Se pudo observar que las preguntas referentes a la frecuencia del peso corporal y al control de la toma de liquidos presentaron menores proporciones de respuestas en el puntaje de la adhesión. Conclusión: el análisis de la asociación entre las variables sociodemográficas y las clínicas y la adbesión de los participantes con insuficiencia cardiaca indicaron una escasa adhesión a la terapia establecida.

Descriptores: Insuficiencia Cardiaca. Terapia. Cooperación del Paciente. Cooperación y Adhesión al Tratamiento. Enfermería.

\section{Introdução}

A insuficiência cardíaca (IC) é definida como uma doença de caráter sistêmico. Ela se expressa pela incapacidade do coração em se encher ou ejetar sangue para atender às necessidades metabólicas e tissulares, ocasionada por desordens estruturais ou funcionais. É considerada a via final da maioria das cardiopatias ${ }^{(1)}$.

No Brasil, a IC constitui-se na primeira causa de internação hospitalar em pacientes acima de 60 anos de idade, sendo associada a elevadas taxas de morbidade e mortalidade. Estima-se que 26 milhões de pessoas são portadoras de IC, e 2 milhões de novos casos são diagnosticados a cada ano no mundo ${ }^{(2)}$.

Nesse contexto, torna-se essencial que as medidas de adesão à terapêutica instituída para IC sejam incorporadas à mudança de estilo de vida necessária para controle da doença. Com esse procedimento é possível alcançar a estabilidade clínica e a manutenção da capacidade funcional, além de reduzir os avanços no remodelamento cardíaco e desfechos clínicos desfavoráveis ${ }^{(1-2)}$.

A adesão terapêutica pode ser definida como a concordância entre o comportamento de um indivíduo e a orientação de um profissional de saúde. Essa anuência refere-se ao uso da medicação, seguimento da dieta, mudança no estilo de vida ou adoção de comportamentos protetores da saúde ${ }^{(3)}$.

No tocante às medidas de adesão terapêutica para pacientes com IC, encontram-se as de manejo farmacológico e não farmacológico. Entre as não farmacológicas, destacam-se a restrição hidrossalina, prática regular de atividade física, verificação diária de peso, reconhecimento precoce de sinais e sintomas de descompensação e uso correto da terapia farmacológica ${ }^{(4)}$.
Evidências na literatura apontam que pacientes com IC têm redução na qualidade de vida em virtude da não adesão às recomendações terapêuticas $^{(2,5-6)}$. Portanto, cabe ao enfermeiro, juntamente com a equipe multidisciplinar e a família do paciente, planejar/executar estratégias que visem melhorar a adesão e, por conseguinte, a qualidade de vida dessa população de risco.

Desse modo, tem-se observado a necessidade de os profissionais de saúde, em especial os enfermeiros, direcionarem pesquisas com o intuito de identificar os fatores que podem contribuir ou dificultar a adesão à terapêutica. É possível subsidiar tecnologias de cuidado com o escopo de reduzir episódios de descompensação, de reinternação e, consequentemente, diminuir os custos do tratamento.

Nesse sentido, a relevância do presente estudo decorre da necessidade de ampliar o estado da arte sobre a temática, incipiente no cenário local e regional. Observou-se que os estudos delineados para avaliar a adesão à terapêutica de pacientes com IC concentram-se nas regiões Sul $^{(7)}$ e Sudeste do país ${ }^{(8-9)}$.

Os objetivos delineados para o estudo foram: analisar a associação entre as variáveis sociodemográficas e clínicas e a adesão de pacientes com IC ao tratamento, e avaliar a adesão desses pacientes ao tratamento.

\section{Método}

Estudo transversal realizado em ambulatório de cardiologia de duas instituições públicas, vinculadas ao Sistema Único de Saúde (SUS) do município de João Pessoa, Paraíba. Este estudo foi realizado entre os meses de outubro de 2017 e março de 2018. 
A população-alvo do estudo envolveu pacientes com diagnóstico médico de IC confirmado em prontuário, atendidos nas instituições selecionadas. Utilizou-se amostragem não probabilística, por conveniência de acordo com o período pré-estabelecido para coleta de dados (cinco meses), levando em consideração a demanda do serviço.

Os critérios de inclusão elegíveis para o estudo foram: pacientes com idade $\geq 18$ anos, de ambos os sexos, em acompanhamento clínico-ambulatorial nas instituições selecionadas.

Os critérios de exclusão foram: pacientes em primeira consulta ambulatorial ou com menos de 48 horas de alta após internação na unidade de clínica médica/cardiológica, com disfunção cognitiva ou que apresentassem barreiras de compreensão e comunicação; além de pacientes oncológicos e em pós-operatório recente (até 60 dias) de qualquer intervenção cirúrgica. Essas condições interferem no comportamento habitual de adesão ao tratamento da IC. Desse modo, a amostra final foi constituída por 50 pacientes.

Para caracterização das variáveis sociodemográficas e clínicas, aplicou-se um instrumento utilizado em estudo prévio em pacientes com $\mathrm{IC}^{(5)}$, como produto das atividades do Grupo de Pesquisa em Doenças Crônicas da Universidade Federal da Paraíba (UFPB), contendo as seguintes variáveis: idade, sexo, escolaridade, situação conjugal e profissional, renda familiar, etiologia da IC, classe funcional segundo os critérios da New York Heart Association (NYHA), presença de comorbidades, fração de ejeção do ventrículo esquerdo (FEVE) contida em laudo de ecocardiograma transtorácico e terapêutica medicamentosa. Adotou-se como FEVE preservada pacientes com FEVE $\geq 50 \%$; e FEVE reduzida, $<50 \%$.

A avaliação da adesão à terapêutica foi obtida por meio de um questionário composto por 10 itens, desenvolvido e validado no Brasil ${ }^{(10)}$. As perguntas incluíram itens relacionados ao uso de medicação, controle de peso, hábitos alimentares, ingestão de líquidos, consumo de bebidas alcoólicas e comparecimento às consultas agendadas. A pontuação total do questionário variou de 0 a 26 pontos; quanto maior a pontuação, mais alta a adesão ${ }^{(10)}$. Embora o questionário não apresentasse ponto de corte definido, adotou-se como adesão satisfatória para análise dos resultados da presente investigação escores $\geq 18$ pontos, índice adotado em investigações prévias desenvolvidas no cenário brasileiro ${ }^{(7,11)}$.

O levantamento das informações foi obtido mediante entrevista estruturada pesquisador/ pesquisado, após a consulta médica, sendo o instrumento de coleta de dados preenchido pelo próprio pesquisador em ambiente reservado.

Os dados obtidos foram inseridos em um banco de dados no Microsoft Excel, contendo um dicionário (CODEBOOK). Em seguida, foram compilados e analisados por meio do programa Statistical Package for Social Science (SPSS) versão 21.0.

Para as variáveis contínuas, calcularam-se média e desvio padrão; para as categóricas, o cálculo foi feito por meio das frequências absoluta e relativa. A normalidade dos dados foi demonstrada utilizando-se o teste de Kolmogorov-Smirnov; a relação das médias do escore de autocuidado com as variáveis com apenas duas categorias foi testada por meio do teste t de Student; às variáveis com três ou mais categorias foi aplicado o teste ANOVA one way; o nível de significância adotado foi de 0,05.

O estudo respeitou as exigências formais contidas nas normas nacionais e internacionais regulamentadoras de pesquisas envolvendo seres humanos. O projeto de pesquisa foi aprovado pelo Comitê de Ética em Pesquisa do Hospital Universitário Lauro Wanderely, com Parecer $n^{\underline{0}}$ 1.255.863 e CAEE: 49420015.9.0000.5183.

\section{Resultados}

Participaram 50 pacientes com IC, com idade média de 59,64士13,07, e variação entre 22 e 85 anos. Observou-se que 56\% pertenciam ao sexo masculino; 66\% eram casados ou viviam em união estável; possuíam média de 4,98ะ3,954 anos de estudo; 68\% tinham etiologia de origem não isquêmica; e 54\% estavam categorizados na classe funcional I. A média da fração de ejeção

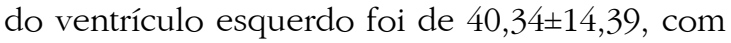
variação de 15 a 69\%. Os medicamentos mais utilizados pelos participantes e registrados em 
prontuário foram diuréticos $40,2 \%$. As características sociodemográficas e clínicas dos participantes do estudo estão apresentadas na Tabela 1.

Tabela 1 - Características sociodemográficas e clínicas de pacientes com insuficiência cardíaca. João Pessoa, Paraíba, Brasil - 2018 (N=50)

\begin{tabular}{|c|c|}
\hline Variáveis & n (\%) \\
\hline \multicolumn{2}{|l|}{ Procedência } \\
\hline João Pessoa & $33(66 \%)$ \\
\hline Outros municípios & $17(34 \%)$ \\
\hline \multicolumn{2}{|l|}{ Sexo } \\
\hline Masculino & $28(56 \%)$ \\
\hline Feminino & $22(44 \%)$ \\
\hline Idade (média \pm desvio padrão) & $59,69 \pm 13,07$ \\
\hline Escolaridade (média \pm desvio padrão) & $4,98 \pm 3,59$ \\
\hline \multicolumn{2}{|l|}{ Cor da pele autodeclarada } \\
\hline Branca & $20(40 \%)$ \\
\hline Não branca & $30(60 \%)$ \\
\hline \multicolumn{2}{|l|}{ Arranjo familiar } \\
\hline Solteiro(a) & $6(12 \%)$ \\
\hline Casado(a)/União estável & $33(66 \%)$ \\
\hline Separado(a)/Divorciado(a) & $6(12 \%)$ \\
\hline Viúvo(a) & $5(10 \%)$ \\
\hline \multicolumn{2}{|l|}{ Situação profissional } \\
\hline Ativo & $2(4 \%)$ \\
\hline Inativo & $48(96 \%)$ \\
\hline \multicolumn{2}{|l|}{ Renda Familiar (salários mínimos) } \\
\hline$<1$ & $2(4 \%)$ \\
\hline 1 a 2 & $43(86 \%)$ \\
\hline$\geq 3$ & $5(10 \%)$ \\
\hline \multicolumn{2}{|l|}{ Etiologia } \\
\hline Isquêmica & $16(32 \%)$ \\
\hline Não isquêmica & $34(68 \%)$ \\
\hline \multicolumn{2}{|l|}{ Classe funcional (New York Heart Association) } \\
\hline I & $13(26 \%)$ \\
\hline II & $27(54 \%)$ \\
\hline III & $10(20 \%)$ \\
\hline Fração de Ejeção do Ventrículo Esquerdo (média \pm desvio padrão) & $40,34 \pm 14,39$ \\
\hline \multicolumn{2}{|l|}{ Terapia Farmacológica } \\
\hline Diuréticos & $41(40,2 \%)$ \\
\hline Betabloqueadores & $37(36,3 \%)$ \\
\hline Anti-hipertensivos & $16(15,7 \%)$ \\
\hline Digitálicos & $6(5,9 \%)$ \\
\hline Antiarrítmicos & $2(2 \%)$ \\
\hline
\end{tabular}

Fonte: Elaboração própria.

No questionário aplicado para avaliar a adesão à terapêutica dos pacientes com IC, observou-se que a média do escore de adesão foi de 13,36 \pm 2,07 pontos, com variação de 8 a 17 pontos, evidenciando-se baixa adesão à terapêutica instituída. Identificou-se que nenhum participante atingiu o ponto de corte estabelecido de adesão satisfatória (escore $\geq 18$ pontos), índice adotado na presente investigação.
A Tabela 2 apresenta a associação das condições sociais com a adesão à terapêutica dos participantes da pesquisa. Com relação aos escores de adesão, verificou-se menor média para os grupos do sexo masculino e ativos economicamente, demonstrando baixa adesão entre as variáveis analisadas. 
Tabela 2 - Associação das condições sociais com adesão à terapêutica de pacientes com insuficiência cardíaca. João Pessoa, Paraíba, Brasil - 2018 (N=50)

\begin{tabular}{|c|c|c|}
\hline Variáveis sociais & Escore de adesão & p-valor \\
\hline \multicolumn{3}{|l|}{ Idade* } \\
\hline$<60$ anos & $13,26 \pm 2,32$ & 0,759 \\
\hline$\geq 60$ anos & $13,44 \pm 1,88$ & \\
\hline \multicolumn{3}{|l|}{ Sexo* } \\
\hline Masculino & $12,96 \pm 2,13$ & 0,131 \\
\hline Feminino & $13,86 \pm 1,93$ & \\
\hline \multicolumn{3}{|l|}{ Raça* } \\
\hline Branca & $13,20 \pm 2,33$ & 0,661 \\
\hline Não Branca & $13,97 \pm 1,92$ & \\
\hline \multicolumn{3}{|l|}{ Estado civil ${ }^{* *}$} \\
\hline Solteiro(a) & $13,33 \pm 1,63$ & \\
\hline Casado(a)/União estável & $13,15 \pm 2,34$ & 0,646 \\
\hline Separado(a)/Divorciado(a) & $13,67 \pm 1,50$ & \\
\hline Viúvo(a) & $14,40 \pm 0,89$ & \\
\hline \multicolumn{3}{|l|}{ Situação profissional* } \\
\hline Ativo & $12,00 \pm 1,41$ & 0,350 \\
\hline Inativo & $13,42 \pm 2,09$ & \\
\hline \multicolumn{3}{|l|}{ Escolaridade* } \\
\hline$<9$ anos & $13,45 \pm 2,16$ & 0,546 \\
\hline$>9$ anos & $13,00 \pm 1,76$ & \\
\hline
\end{tabular}

Fonte: Elaboração própria.

* teste $t$ - student;

** teste Anova one way

A Tabela 3 apresenta a associação das condições clínicas com a medida de adesão. Observou-se que os pacientes com classe funcional
III e com > 1 comorbidade, apresentaram menores escores, demonstrando baixa adesão ao tratamento.

Tabela 3 - Associação das condições clínicas com adesão à terapêutica de pacientes com insuficiência cardíaca. João Pessoa, Paraíba, Brasil - 2018 (N=50)

\begin{tabular}{|c|c|c|}
\hline Variáveis clínicas & Escore de adesão & p-valor \\
\hline \multicolumn{3}{|l|}{ Etiologia* } \\
\hline Isquêmica & $13,44 \pm 2,33$ & \multirow[t]{2}{*}{0,859} \\
\hline Não isquêmica & $13,32 \pm 1,98$ & \\
\hline \multicolumn{3}{|c|}{ New York Heart Association ${ }^{* *}$} \\
\hline Classe I & $13,62 \pm 1,75$ & \multirow{3}{*}{0,142} \\
\hline Classe II & $13,67 \pm 2,07$ & \\
\hline Classe III & $13,20 \pm 2,25$ & \\
\hline \multicolumn{3}{|l|}{ Comorbidades* } \\
\hline 1 comorbidade & $13,53 \pm 2,18$ & \multirow[t]{2}{*}{0,443} \\
\hline$>1$ comorbidade & $13,06 \pm 1,89$ & \\
\hline \multicolumn{3}{|c|}{ Fração de Ejeção de Ventrículo Esquerdo* } \\
\hline Reduzida & $13,13 \pm 2,20$ & \multirow[t]{2}{*}{0,585} \\
\hline Preservada & $13,55 \pm 1,86$ & \\
\hline
\end{tabular}

Fonte: Elaboração própria.

*teste $t$ - student.

** teste Anova one way. 
Adicionalmente, procedeu-se a análise da frequência de respostas para cada variável que compõe o escore de adesão ao tratamento, conforme ilustra a Tabela 4. Observa-se que as questões alusivas à frequência do peso corporal (Q2) e controle na ingestão de líquidos (Q6; Q7; Q8) apresentaram as menores proporções de respostas, evidenciado baixa adesão a essas recomendações. Nota-se que as questões referentes ao uso de medicamentos conforme prescrição (Q1), a não ingestão de bebidas alcoólicas (Q9) e o seguimento de consultas e exames agendados (Q10) exibiriam as melhores frequências de respostas equivalentes a uma adesão satisfatória entre as medidas instituídas para o tratamento da IC.

Tabela 4 - Distribuição de frequência de respostas segundo itens do instrumento de adesão ao tratamento. João Pessoa, Paraíba, Brasil - 2018 (N=50)

\begin{tabular}{lcc}
\hline Itens & Resposta & n (\%) \\
\hline $\begin{array}{l}\text { 1. Usou os medicamentos nos últimos 15 dias, de } \\
\text { acordo com a prescrição médica? }\end{array}$ & Sempre $(94 \%)$ \\
$\begin{array}{l}\text { 2. Com que frequência você se pesa? } \\
\text { 3. Você coloca sal nos seus alimentos? }\end{array}$ & Não verifico & $37(74 \%)$ \\
$\begin{array}{l}\text { 4. Você adiciona tempero pronto, tais como caldo de } \\
\text { galinha Sazon no preparo dos alimentos? }\end{array}$ & No cozimento dos alimentos \\
$\begin{array}{l}\text { 5. Você faz refeições ou come alimentos fora do lar } \\
\text { sem restrição de sal? }\end{array}$ & Não uso (Nada) \\
$\begin{array}{l}\text { 6. Na quantidade de líquido diário recomendado, você } \\
\text { considera também sopas, sorvete, gelatina, suco, leite, } \\
\text { chá, café e bebidas não alcoólicas? }\end{array}$ & Nunca \\
$\begin{array}{l}\text { 7. Você diminuiu a ingestão de líquidos de acordo } \\
\text { com a instrução do seu médico ou enfermeiro? }\end{array}$ & Nunca \\
$\begin{array}{l}\text { 8. Você considera o líquido (caldo) de frutas na } \\
\text { quantidade diária de líquido que foi recomendada }\end{array}$ & \\
$\begin{array}{l}\text { a você, tais como laranjas, melão, melancia, abacaxi, } \\
\text { água de coco, bergamota etc.? } \\
\text { 9. Você ingere alguma bebida alcoólica? } \\
\begin{array}{l}\text { 10. Você faltou a alguma consulta médica ou exame } \\
\text { agendado nos últimos 15 dias? }\end{array}\end{array}$ & Nunca \\
\hline
\end{tabular}

Fonte: Elaboração própria.

\section{Discussão}

Analisar a adesão à terapêutica instituída e verificar a associação de condições sociais e clínicas de pacientes com IC, configura-se como uma estratégia para identificar possíveis fatores que possam comprometer o plano terapêutico instituído e a sobrevida dessa população em face às crises de descompensação clínica.

O presente estudo revelou relativo equilíbrio de distribuição entre homens e mulheres, com maioria autodeclarada da raça branca, casada ou vivendo em união estável, com baixo nível de escolaridade, inativos economicamente e baixa renda familiar. Clinicamente, prevaleceu a IC de etiologia não isquêmica, classe funcional I, com comorbidades associadas e fração de ejeção de ventrículo esquerdo diminuída. Esses dados são similares ao perfil de pacientes acometidos por IC no contexto nacional ${ }^{(8-11)}$.

Concernente às condições sociais, pôde-se observar médias de escore de adesão menor para o sexo masculino. Embora não se encontre evidência consistente sobre o comportamento da adesão entre os sexos, este achado pode ser explicado pela maior prevalência de fatores de risco associados à IC, como dislipidemias, tabagismo e elitismo em pessoas do sexo masculino, 
o que pode favorecer a baixa adesão às recomendações no manejo clínico da doença ${ }^{(1)}$.

Constatou-se que a maioria dos pacientes que se declararam casados ou em união estável apresentaram menores escores quando comparados aos demais. Este dado chama atenção, uma vez que o apoio familiar é considerado de extrema importância para a recuperação e manutenção do estado clínico e da estabilidade dos pacientes com IC ${ }^{(12)}$.

Nesse aspecto, presume-se que um relacionamento familiar conflituoso pode comprometer a adesão ao regime terapêutico. Por isso, antes de propor estratégias de envolvimento da família no cuidado ao paciente com IC, sugere-se que os profissionais de saúde verifiquem o suporte familiar que os pacientes dispõem. Entretanto, ressalta-se que o apoio familiar é indispensável para a motivação de ações de autocuidado, que são essenciais para a manutenção da saúde em pacientes acometidos por doenças crônicas ${ }^{(12)}$.

Concordante com essa assertiva, estudo conduzido em duas clínicas especializadas para pacientes com IC crônica, acompanhados por enfermeiros, demonstrou que aqueles que viviam com suas famílias tiveram escores de adesão mais elevados ${ }^{(9)}$. Assim, recomenda-se que intervenções de enfermagem sejam conduzidas, a fim de envolver os familiares nas estratégias de cuidado. O propósito disso é fortalecer elos para a melhor capacidade de enfrentamento dos sintomas físicos e psicológicos inerentes à doença, bem como propiciar a adesão terapêutica e o bem-estar.

Em relação à escolaridade, os achados apresentaram-se homogêneos. Não houve diferença nos escores quando comparados pela média de anos de estudo. Contudo, espera-se que pessoas com melhores níveis de escolaridade apresentem maior compreensão da doença, bem como melhor acesso aos cuidados de saúde e, por conseguinte, melhor adesão ao tratamento.

De acordo com essa afirmativa, estudo conduzido com 82 pacientes com IC descompensada encontrou correlação significativa entre autocuidado e escolaridade, evidenciando que o maior tempo de estudo melhora a gestão para o autocuidado $^{(13)}$.

Destacou-se, na amostra, elevado número de pessoas economicamente inativas. Acredita-se que a baixa adesão à terapêutica evidenciada pode ter contribuído para a exacerbação de sintomas e limitações físicas inerentes à IC, ocasionando aposentadorias precoces e, por conseguinte, ônus ao sistema financeiro público, já que a média de idade dos entrevistados encontra-se abaixo da idade estabelecida pelo órgão de seguridade social no país.

Considerando as condições clínicas, observou-se que os investigados em classe funcional III e com fração de ejeção reduzida apresentaram menores escores de adesão à terapêutica. Sabe-se que pacientes que apresentam essas condições clínicas são caracterizados com sintomas desencadeados em atividades menos intensas que as cotidianas ou em pequenos esforços ${ }^{(6)}$. Entretanto, os níveis de adesão foram baixos para as três classes funcionais identificadas no estudo. São achados considerados preocupantes, tendo em vista que os pacientes que apresentavam ausência de sintomatologia mostraram-se poucos aderentes à terapêutica.

Nesse sentido, o estudo aponta a necessidade de identificar quais as dificuldades para se efetivar as medidas de autocuidado essenciais para a manutenção da estabilidade clínica. Desse modo, sugere-se que medidas educativas sistemáticas em saúde, baseadas em evidências científicas, sejam conduzidas para minimizar progressões no remodelamento cardíaco pela falta de adesão à terapêutica. Ademais, é imperioso o empoderamento dessa clientela para a execução das medidas de autocuidado essenciais à saúde.

No presente estudo, constatou-se baixa adesão na avaliação da terapêutica instituída, isto é, ausência de participantes com adesão satisfatória (escore $\geq 18$ pontos). Os resultados encontrados são similares aos de pesquisas desenvolvidas no cenário nacional ${ }^{(7,9)}$. Outro estudo realizado na Colômbia - cujo objetivo foi determinar a prevalência de adesão ao tratamento farmacológico e não farmacológico de pacientes com IC - evidenciou que $80,2 \%$ dos 
participantes apresentaram-se como frequentemente aderentes, e que apenas 3,73\% foram aderentes ao tratamento ${ }^{(14)}$.

Estudo com abordagem qualitativa, cujo objetivo foi identificar as crenças relacionadas aos fatores que dificultavam a adesão ao tratamento em pacientes com IC, evidenciou a falta de acesso ao serviço médico, demora na marcação de consultas e dificuldades no transporte/ locomoção ao serviço de saúde como fatores principais da baixa adesão à terapêutica ${ }^{(8)}$.

Outra pesquisa, longitudinal e conduzida em cinco regiões brasileiras, identificou que 63,7\% dos pacientes com IC descompensada receberam orientações na alta hospitalar sobre a correta tomada da terapia medicamentosa. Apenas 34,9\% e $16,2 \%$ foram orientados a, respectivamente, seguir uma dieta com baixo teor de sódio e a praticar atividade física de modo regular. Estas recomendações são essenciais para a manutenção da estabilidade clínica e a prevenção de reinternações hospitalares indesejadas ${ }^{(1)}$.

Esses achados remetem às reflexões sobre a assistência oferecida, tornando-se um desafio para as equipes de saúde e pacientes incorporarem, ao estilo de vida, as medidas de adesão farmacológicas e não farmacológicas necessárias para manutenção da capacidade funcional e estabilidade clínica. Muitas dessas situações necessitam de rupturas de paradigmas culturais e sociais.

Além disso, soma-se a ausência de políticas públicas de saúde específicas para o seguimento dessa clientela, ficando a atenção especializada responsável pelo gerenciamento dos cuidados de saúde, ocasionando retardo no diagnóstico e no início da abordagem terapêutica. Desse modo, aponta-se a reorganização da rede de atenção em saúde como possível estratégia para o fortalecimento do cuidado de pacientes com IC.

Os resultados encontrados na presente investigação foram inferiores à média de escore de estudo realizado em duas regiões do país, com 340 pacientes, que foi de $16 \pm 4$ pontos. Os autores afirmam que apenas 36,5\% dos pacientes apresentaram taxa de adesão igual ou superior ao ponto de corte estabelecido $(\geq 18 \text { pontos })^{(9)}$.
Em relação à proporção das respostas das variáveis que compõem o escore de adesão ao tratamento, identificou-se que o controle do peso corporal e a ingestão de líquidos foram as recomendações menos aderentes. Esses dados corroboram o estudo multicêntrico brasileiro que evidenciou, entre as medidas não farmacológicas, que a restrição de líquido foi a menos executada $^{(15)}$.

Sabe-se que o paciente com IC deve ser orientado a restringir a quantidade diária de líquidos em torno de 1.000 a $1.500 \mathrm{ml}$. No entanto, essa recomendação deverá ser realizada de acordo com as condições clínicas e a terapêutica medicamentosa utilizada, em especial o uso de diuréticos ${ }^{(16)}$.

Adicionalmente, identificou-se que o controle do peso corporal é outra recomendação com baixa adesão, e tem como finalidade monitorar o surgimento de sinais e sintomas de descompensação clínica, a exemplo do acúmulo de líquidos no corpo. Nesse sentido, essas medidas são instituídas, a fim de direcionar a terapêutica farmacológica. Torna-se imperativo que essas medidas sejam clarificadas para o público-alvo. Isso pode ocorrer por meio de uma educação sistemática subsidiada por metodologias problematizadoras, objetivando favorecer a adesão e minimizar prognósticos negativos.

Em contrapartida, evidenciou-se o uso da medicação conforme prescrição, a não ingestão de bebidas alcoólicas e o seguimento das consultas e exames agendados, como as medidas de adesão ao tratamento com maiores frequências de respostas satisfatórias. No entanto, esses resultados levantam a hipótese de que, embora os usuários compareçam às consultas previamente agendadas, presume-se que existem lacunas que necessitam ser dirimidas. Como exemplo, cita-se a falta de conhecimento/entendimento da doença, a relação profissional/paciente ou fatores externos que influenciam a não adesão à terapêutica instituída.

Em relação à restrição do consumo de sal na dieta, identificou-se menor proporção de pacientes que se referiram à realização da restrição salina. Essa recomendação tem sido estudada 
pela literatura, e as evidências encontradas apontam baixa adesão a uma dieta com baixo teor de sódio em pacientes com IC ${ }^{(16-17)}$.

Concordante como essa assertiva, pesquisa conduzida no Canadá, com 237 pacientes, concluiu que o autorrelato de adesão a uma dieta com baixo teor de sódio apresenta baixa confiabilidade, uma vez que pacientes com IC associam a ideia de seguir uma dieta com baixo teor de sódio principalmente com o não uso de sal para o preparo das refeições ou seu uso na mesa, e não com a redução da frequência de ingestão de alimentos processados com alto teor de sódio ${ }^{(17)}$.

É importante ressaltar que se observou, durante o desenvolvimento da pesquisa, que os participantes não foram acompanhados por enfermeiros, tampouco por equipe multidisciplinar integrada no cuidado especializado ao paciente com IC. No entanto, entende-se que o enfermeiro, como membro da equipe de saúde, configura-se como um facilitador do processo educativo necessário para a compreensão do processo saúde/doença ${ }^{(15)}$. Além disso, intervenções de enfermagem vêm sendo utilizadas para efetivar as medidas de manejo não farmacológico, primordiais para minimizar exacerbações de sintomas clínicos e fomentar o autocuidado.

Evidências apontam o papel de destaque desempenhado pelo enfermeiro na abordagem terapêutica ao paciente com IC. Corrobora essa afirmativa estudo realizado com 32 pacientes cujo objetivo foi verificar a adesão ao tratamento de pacientes com IC após alta hospitalar -, que evidenciou, por meio de visitas domiciliares de cunho educativo realizadas por enfermeiros, o aumento significativo dos escores de adesão, passando de $16,0 \pm 2,6$ pontos na primeira visita para o escore $20,4 \pm 2,7$ pontos na terceira visita $(\mathrm{p}=0,001)^{(7)}$.

Desse modo, fica evidente a necessidade da implementação de programas de acompanhamento de pacientes com IC por enfermeiros, em especial na atenção primária de saúde, uma vez que estratégias como visitas domiciliares ${ }^{(11)}$ e monitorização telefônica ${ }^{(18)}$ mostram-se como ferramentas de cuidado de baixo custo e apresentam resultados satisfatórios para o sucesso da terapêutica instituída, sobrevida e melhor percepção da qualidade de vida. Dessa maneira, sugere-se que tecnologias de cuidado, com base no contexto local, sejam delineadas com o fito de dirimir eventuais lacunas de conhecimento que exerçam impacto negativo na adesão ao tratamento.

Ressalta-se que os resultados encontrados são limitados pelo delineamento transversal, o que impede de estabelecer relações de causa-efeito. Sugere-se que estudos longitudinais sejam conduzidos com amostras maiores, a fim de comparar grupos por sexo, e com o escopo de ratificar ou refutar as evidências suscitadas na presente investigação. Esclarece-se também que os achados evidenciados não podem ser generalizados em razão da medida de adesão ao tratamento ser autorreferida. Em acréscimo, pode-se apontar o tamanho amostral, que poderia ter sido de maior amplitude em face dos problemas operacionais no serviço investigado, que acabou por afetar a coleta de dados e pode ter influenciado a ausência de resultados estatísticos significativos. Nessa perspectiva, sugere-se que investigações futuras, prospectivas e baseadas em teoria comportamentais, sejam conduzidas, a fim de explorar os fatores determinantes do comportamento de adesão à terapêutica de pacientes com IC.

\section{Conclusão}

Os resultados revelaram que os participantes apresentaram baixa adesão à terapêutica instituída. Pacientes do sexo masculino, ativos economicamente, em classe funcional III e com $>1$ comorbidade associada à IC demonstraram menores escores de adesão. As medidas de manejo não farmacológico, como controle do peso corporal e restrição na ingestão de líquidos, exibiram menor proporção de adesão entre os pacientes avaliados. O uso dos medicamentos prescritos, a não ingestão de bebidas alcoólicas e o seguimento de consultas e exames agendados exibiriam as melhores frequências de resposta, indicando uma adesão satisfatória entre as medidas instituídas para manejo terapêutico da IC. 
Para a prática de enfermagem, os resultados deste estudo apontam para a necessidade de implementar intervenções, como consultas de enfermagem, visitas domiciliares e monitorização remota, a fim de potencializar a adesão à terapêutica e, por conseguinte, favorecer a redução nos índices de morbimortalidade dessa população.

\section{Colaborações:}

1 - projeto, análise e interpretação dos dados: Mailson Marques de Sousa e Simone Helena dos Santos Oliveira;

2 - redação do artigo e revisão crítica relevante do conteúdo intelectual: Mailson Marques de Sousa, Rhaira Pereira Campos, Jacira dos Santos Oliveira e Simone Helena dos Santos Oliveira;

3 - aprovação final da versão a ser publicada: Mailson Marques de Sousa e Simone Helena dos Santos Oliveira.

\section{Referências}

1. Albuquerque DC, Souza Neto JD, Bacal F, Rohde LEP, Bernardez-Pereira S, Berwanger O, et al. I Brazilian Registry of Heart Failure - Clinical Aspects, Care Quality and Hospitalization Outcomes. Arq Bras Cardiol. 2015 Jun;104(6):43342. doi: 10.5935/abc. 20150031

2. Mesquita EV, Jorge AJL, Rabelo LM, Souza Jr CV. Understanding ospitalization in patients with Heart Failure. Int J Cardiovasc Sci. 2017;30(1):81-90. DOI: $10.5935 / 2359-4802.20160060$

3. Organización Mundial de La Salud. Adherencia a los tratamientos a largo plazo: Pruebas para la acción. Genebra; 2004.

4. Jaarsma T, Stromberg A, Gal TB, Cameron J, Driscoll A, Duengen HD, et al. Comparison of self-care behaviors of heart failure patients in 15 countries worldwide. Patient Educ Couns. 2013;92(1):114-20. DOI:10.1016/j.pec.2013.02.017.

5. Sousa MM, Oliveira JS, Soares MJGO, Bezerra SMMS, Araújo AA, Oliveira SHS. Association of social and clinical conditions to the quality of life of patients with heart failure. Rev Gaúcha Enferm. 2017;38(2):e65885. DOI: 10.1590/1983-1447.2017.02.65885
6. Hoekstra T, Jaarsma T, van Veldhuisen DJ, Hillege HL, Sanderman R, Lesman-Leegte I. Quality of life and survival in patients with heart failure. Eur J Heart Fail. 2013;15(1):94-102. DOI: 10.1093/ EURJHF/HFS148

7. Mantovani VM, Ruschel KB, Souza EM, Mussi C, Silva ERR. Treatment adherence in patients with heart failure receiving nurse-assisted home visits. Acta Paul Enferm. 2015;28(1):41-7 DOI: 10.1590/1982-0194201500008

8. Lucas TC, Stuchi RAG, Arreguy-Sena C, Cordeiro AF. Heart failure and difficulties in treatment adherence. [Internet] 2017;7:e1871. DOI: https:// doi.org/10.19175/recom.v7i0.1871.

9. Silva AF, Cavalcanti ACD, Malta M, Arruda CS, Gandin T, Fé A, et al. Treatment adherence in heart failure patients followed up by nurses in two specialized clinics. Rev Latino-Am Enfermagem. 2015;23(5):888-94. DOI: http:// dx.doi.org/10.1590/0104-1169.0268.2628

10. Bocchi EA, Cruz F, Guimarães G, Pinho Moreira LF, Issa VS, Ayub Ferreira SM. Longterm prospective, randomized, controlled study using repetitive education at six-month intervals and monitoring for adherence in heart failure outpatients: the REMADHE trial. Circ Heart Fail. 2008;1(2):115-24. DOI: http://dx.doi.org/10.1161/ CIRCHEARTFAILURE.107.744870

11. Mussi CM, Ruschel K, Nogueira A, Lopes M, Trojahn MM, Paraboni CC, et al. Visita domiciliar melhora conhecimento, autocuidado e adesão na insuficiência cardíaca: ensaio clínico randomizado HELEN-I. Rev Latino-Am Enfermagem. 2013;21:1-9 DOI: http://dx.doi.org/10.1590/S0104$-11692013000700004$

12. Saldaña DMA, Sánchez AR, Castro NMT, Mora ALB, Beltrán NXN, Florez PD. Skills of family caretakers to care for patients with heart failure. Rev Cuid. 2017;8(3):1721-32. DOI: http://dx.doi. org/10.15649/cuidarte.v8i3.407

13. Linn AC, Azollin K, Souza EM. Association between selfcare and hospital readmissions of patients with heartFailure. RevBrasEnferm. 2016;69(3):500-6.doi: http://dx.doi.org/10.1590/0034-7167.2016690312i

14. Rojas Sánchez LZ, Echeverría Correa LE, Camargo Figuera FA. Adherence to phararmacological and non-pharmacological treatment in patients with heart failure. Enferm glob [Internet]. 2014 [cited 2018 Jun 11];13(36):15-25. Available from: http:// scielo.isciii.es/pdf/eg/v13n36/en_clinica1.pdf 
Mailson Marques de Sousa, Rhaira Pereira Campos, Jacira dos Santos Oliveira, Simone Helena dos Santos Oliveira

15. Rabelo ER, Aliti GB, Costa Linch GFC, Sauer JM, Mello AMFS, Martins SM, et al. Non-pharmacological management of patients with decompensated heart failure: a multicenter study - EMBRACE. Acta Paul Enferm. 2012;25(5):660-5. DOI: http://dx.doi. org/10.1590/S0103-21002012000500003

16. Philipson H, Ekman I, Forslund HB, Swedberg K, Schaufelberger M. Salt and fluid restriction is effective in patients with chronic heart failure. Eur J Heart Fail. 2013;15(11):1304-10. DOI: https://doi. org/10.1093/eurjhf/hft097

17. Colin-Ramirez E, McAlister FA, Woo E, Wong N, Ezekowitz JA. Association between self-reported adherence to a low-sodium diet and dietary habits related to sodium intake in heart failure patients. J Cardiovasc Nurs. 2015;30(1):58-65 DOI: http:// dx.doi.org/10.1097/JCN.0000000000000124

18. Bashi N, Karunanithi M, Fatehi F, Ding $\mathrm{H}$, Walters D. Remote Monitoring of Patients With Heart Failure: An Overview of Systematic Reviews. J Med Internet Res. 2017;20;19(1):e18. DOI: 10.2196/jmir.6571

Recebido: 20 de março de 2019

Aprovado: 9 de setembro de 2019

Publicado: 14 de novembro de 2019

A Revista Baiana de Enfermagem utiliza a Licença Creative Commons - Atribuição-NãoComercial 4.0 Internacional. https://creativecommons.org/licenses/by-nc/4.0/

Este artigo é de acesso aberto distribuído sob os termos da Licença Creative Commons (CC BY-NC). Esta licença permite que outros remixem, adaptem e criem a partir do seu trabalho para fins não comerciais. Embora os novos trabalhos tenham de lhe atribuir o devido crédito e não possam ser usados para fins comerciais, os usuários não têm de licenciar esses trabalhos derivados sob os mesmos termos. 